Research Article

\title{
Finite-Horizon H-Infinity Control for Time-Varying State-Saturated Systems under Stochastic Communication Protocol
}

\author{
Kewang Huang $\mathbb{1 D}^{1}$ and Feng Pan $^{2}$ \\ ${ }^{1}$ Wuxi Institute of Technology, Wuxi 214121, Jiangsu, China \\ ${ }^{2}$ Key Laboratory of Advanced Process Control for Light Industry, Ministry of Education, Jiangnan University, Wuxi 214122, \\ Jiangsu, China
}

Correspondence should be addressed to Kewang Huang; huangkw@wxit.edu.cn

Received 9 April 2021; Accepted 7 October 2021; Published 10 November 2021

Academic Editor: Yuqiang Wu

Copyright (C) 2021 Kewang Huang and Feng Pan. This is an open access article distributed under the Creative Commons Attribution License, which permits unrestricted use, distribution, and reproduction in any medium, provided the original work is properly cited.

\begin{abstract}
In this paper, the Ho control problem is investigated for a class of time-varying state-saturated systems with both stochastic parameters and nonlinearities under the stochastic communication protocol (SCP). The sensor-to-controller network is considered where only one sensor can obtain access to the communication network at each transmission instant. The SCP is adopted to mitigate the undesirable data collision phenomenon. The model transformation technique is employed to simplify the addressed problem, and then, the completing squares method is carried out to obtain a sufficient condition for the existence of the finite-horizon $\mathrm{H} \infty$ controller. The controller parameters are characterized by solving two coupled backward recursive Riccati-like difference equations. A simulation example is finally utilized to illustrate the effectiveness of the proposed controller design scheme.
\end{abstract}

\section{Introduction}

Due to its advantages of easy sharing, strong flexibility, and easy installation and maintenance, networked control systems (NCSs) have attracted a large number of scholars' attention in recent years [1-4].

Most existing methods of the controller and the filter design are only for infinite time-invariant controlled object. In the actual NCSs, due to the operating point offset, equipment aging, and environmental factors, many systems are time varying. Therefore, it is of more practical significance to study the transient characteristics of time-varying systems in the finite horizon than the steady-state characteristics of timeinvariant systems. The corresponding fuzzy control problem, finite-time domain filtering problem, and envelope constraint problem for continuous time-varying system, discrete timevarying system, and discrete time-varying nonlinear system are discussed in [5-7], respectively.
In NCSs, the bandwidth of communication network is limited, which tends to cause data conflicts of control or measurement signals in the process of network transmission, resulting in packet loss and network-induced delay $[8,9]$. If multiple nodes in the network attempt to simultaneously transfer data, these phenomena caused by nonideal networks may be even more serious. Therefore, an effective way to avoid data conflicts is to join the communication protocol to manage the rights of each node to access the network. Random communication protocols have been widely studied and applied for their advantages of random access and strong expansibility $[10,11]$. In [12], the filtering problem of a kind of linear time-invariant system under a random communication protocol was studied, which described the communication process between the sensor and the filter as a Markov chain. In [13], the auxiliary vector and a new state estimation structure were proposed to the problem of finitetime state estimation of delayed artificial neural network 
under the random communication protocol. In [14], a random pulse delay model was proposed under the consideration of packet loss, network-induced delay, and random communication protocol. Furthermore, an output feedback controller was designed to ensure the stability of the system in the mean square sense. In $[15,16]$, the sliding mode control and robust finite-time stability problem with time-varying delays were investigated.

It is important to note that the above literature implicitly assumes that the system state is not saturated. However, in practice, due to the physical limitations or protection measures of the equipment, state saturation often occurs. For example, mechanical systems with position and speed limits, digital filters implemented in finite word length formats, and neural networks with saturated transfer functions $[17,18]$. In these cases, the state of the system is constrained within a bounded set. If state saturation is not considered in the comprehensive analysis, the desired performance of the closed-loop system cannot be guaranteed. Although the control and filtering of NCSs in infinite time domain and random communication protocol have been studied in depth, the control problem in finite-time domain of state-saturated time-varying system has not attracted attention under the influence of random communication protocol, which arouses the author's research interest.

In summary, this paper is concerned with the control problem of the state saturation system in the finite-time domain based on the random communication protocol and considering the influence of random parameters and random nonlinearity. The main contributions in this paper are as follows: (1) both stochastic parameters and nonlinearities under the stochastic communication protocol are considered in the design of the output feedback controllers guaranteeing the desired $H_{\infty}$ performance, (2) a sufficient condition is provided for an auxiliary index which is closely related to the desired finite-horizon $H_{\infty}$ performance, and (3) a suboptimal controller design scheme is provided by solving two coupled backward recursive Riccati-like difference equations.

The rest of this paper is organized as follows. Section 2 introduces the problem description. Sufficient conditions for the $H_{\infty}$ performance analysis and controller design are given in Sections 3 and 4. In Section 5, a numerical example is presented. Finally, Section 6 concludes the paper.

Notations: the notations used in this paper are as follows. Rn denotes the $n$-dimensional Euclidean space. $\mathscr{L}_{2[0, N]}$ is the space of square summable vectors. $E\{\cdot\}$ stands for the mathematical expectation and Prob $\{\cdot\}$ means the occurrence probability of the event. In symmetric block matrices or complex matrix expressions, we use $*$ to represent a term that is induced by symmetry and diag\{.\} denotes the block diagonal matrix. If their dimensions are not explicitly stated, they are assumed to be compatible for algebraic operations. The notation $\mathbf{P}>0(\geq 0)$ means that $\mathbf{P}$ is a symmetric and positive definite (semidefinite) matrix. 0 and $\mathbf{I}$ represent the zero matrix and identity matrix with compatible dimensions.

\section{Problem Description}

Consider the state-saturated random time-varying system in the finite horizon $k \in[0, N]$ :

$$
\left\{\begin{array}{l}
\mathbf{x}_{k+1}=\sigma\left(\mathbf{A}_{k}\left(\mu_{k}\right) \mathbf{x}_{k}+\mathbf{f}\left(\mathbf{x}_{k}\right)+\mathbf{B}_{k} \mathbf{u}_{k}+\mathbf{D}_{k} \mathbf{w}_{k}\right) \\
\mathbf{y}_{k}=\mathbf{C}_{k} \mathbf{x}_{k}+\mathbf{E}_{k} \mathbf{v}_{k} \\
\mathbf{z}_{k}=\mathbf{L}_{k} \mathbf{x}_{k}
\end{array}\right.
$$

where $\mathbf{x}_{k} \in \mathbb{R}^{n_{x}}, \mathbf{u}_{k} \in \mathbb{R}^{n_{u}}, \mathbf{z}_{k} \in \mathbb{R}^{n_{z}}$, and $\mathbf{y}_{k} \in \mathbb{R}^{n_{y}}$, respectively, represent system state, control input, controlled output, and measured output vector before transmission from the sensor-to-controller network. $\mathbf{w}_{k} \in \mathscr{L}_{2[0, N]}$ and $\mathbf{v}_{k} \in \mathscr{L}_{2[0, N]}$ are process noise and measurement interference of the system, respectively. The matrices $\mathbf{B}_{k}, \mathbf{D}_{k}, \mathbf{C}_{k}, \mathbf{E}_{k}$, and $\mathbf{L}_{k}$ are time-varying parameter matrices with known appropriate dimensions. $\sigma(\cdot)$ is a saturation function, $\mathbf{A}_{k}\left(\mu_{k}\right)$ is a random parameter matrix, $\mu_{k}$ is a zero-mean Gaussian white noise sequence with variance 1 , and $\mathbf{f}\left(\mathbf{x}_{k}\right)$ is a random nonlinear function of the system. The random parameter matrix $\mathbf{A}_{k}\left(\mu_{k}\right)$ and the random nonlinear function $\mathbf{f}\left(\mathbf{x}_{k}\right)$ are independent of each other.

As [19] shows, the random parameter matrix $\mathbf{A}_{k}\left(\mu_{k}\right)$ has the following statistical characteristics:

$$
\begin{aligned}
E\left\{\mathbf{A}_{k}\left(\mu_{k}\right)\right\} & =\bar{A}_{k}, \\
\operatorname{Cov}\left\{a_{m u}^{k}, a_{s t}^{k}\right\} & =T_{a_{m u}^{k}, a_{s t}^{k},}
\end{aligned}
$$

where $T_{a_{m u}^{k}, a_{s t}^{k}}$ is a known parameter and $a_{s t}^{k}$ is $(s, t)$ th element of matrix $\mathbf{A}_{k}\left(\mu_{k}\right)$, i.e., $a_{s t}^{k}=\mathbf{A}_{k}\left(\mu_{k}\right)_{[s, t]}$.

For all $\mathbf{x}_{k}$, the first moment and covariance of the random nonlinear function $\mathbf{f}\left(\mathbf{x}_{k}\right)$ satisfy

$$
\begin{aligned}
E\left\{\mathbf{f}\left(\mathbf{x}_{k}\right) \mid \mathbf{x}_{k}\right\} & =0, \\
E\left\{\mathbf{f}\left(\mathbf{x}_{k}\right) \mathbf{f}^{T}\left(\mathbf{x}_{j}\right) \mid \mathbf{x}_{k}\right\} & =0, \quad k \neq j, \\
E\left\{\mathbf{f}\left(\mathbf{x}_{k}\right) \mathbf{f}^{T}\left(\mathbf{x}_{k}\right) \mid \mathbf{x}_{k}\right\} & =\sum_{n=1}^{q} \ell_{n, k} \ell_{n, k}^{T} x_{k}^{T} \Gamma_{n, k} \mathbf{x}_{k},
\end{aligned}
$$

where $q$ is a known nonnegative integer and $\ell_{n, k}$ and $\Gamma_{n, k}(n=$ $1,2, \ldots, q)$ are known appropriate dimension matrices.

Define saturation function $\sigma(\cdot): \mathbb{R}^{n_{x}} \longrightarrow \mathbb{R}^{n_{x}}$ :

$$
\begin{aligned}
\sigma\left(\mathbf{x}_{k}\right) & =\left[\begin{array}{llll}
\sigma_{1}\left(\mathbf{x}_{k}^{1}\right) & \sigma_{2}\left(\mathbf{x}_{k}^{2}\right) & \cdots & \sigma_{n_{x}}\left(\mathbf{x}_{k}^{n_{x}}\right)
\end{array}\right]^{T}, \\
\sigma_{m}\left(\mathbf{x}_{k}^{m}\right) & =\operatorname{sign}\left(\mathbf{x}_{k}^{m}\right) \min \left\{\left|\mathbf{x}_{k}^{m}\right|, \mathbf{x}_{k, \max }^{m}\right\}, \quad m=1,2, \ldots, n_{x},
\end{aligned}
$$

where $\mathbf{x}_{k}^{m}$ is the component of vector $\mathbf{x}_{k}$ and $\mathbf{x}_{k, \max }^{m}>0$ is the saturation level.

In order to solve the problem of network congestion and data conflict caused by multiple transmission nodes using the communication network at the same time, random communication protocol is adopted in the network. Under the SCP scheduling, only one node is allowed to access the network and transmit data at each transport moment. $\xi_{k}$ is defined as the node that obtains access to the network at the 
instant $k$, and its value is determined by the Markov chain. The transition probability matrix is $P_{k}=\left[p_{k}^{i j}\right]_{n_{y} \times n_{y}}$, specifically defined as

$$
p_{k}^{i j}=\operatorname{Prob}\left\{\xi_{k+1}=j \mid \xi_{k}=i\right\},
$$

where $p_{k}^{i j} \in[0,1]\left(i, j \in\left\{1,2, \ldots, n_{y}\right\}\right)$ represents the transition probability of $k$ moment from state $i$ to state $j$ and satisfies $\sum_{j=1}^{n_{y}} p_{k}^{i j}=1$.

Set the measurement output after transmission from the sensor-to-controller network as $\bar{y}_{k}=\left[\bar{y}_{1, k} \bar{y}_{2, k} \cdot \bar{y}_{n_{y}, k}\right]^{T}$, and $\bar{y}_{i, k}$ represents the measurement output of the $i$ sensor node received by the controller at the transmission time $k$. Zero input processing strategy is adopted, and the updating rules of $\bar{y}_{i, k}$ are

$$
\bar{y}_{i, k}= \begin{cases}\mathbf{y}_{i, k}, & \text { if } i=\xi_{k} \\ 0, & \text { Others. }\end{cases}
$$

On the basis of formula (9), there are

$$
\bar{y}_{k}=\Phi_{\xi_{k}} \mathbf{y}_{k}
$$

where $\Phi_{\xi_{k}}=\operatorname{diag}\left\{\delta\left(\xi_{k}-1\right), \delta\left(\xi_{k}-2\right), \ldots, \delta\left(\xi_{k}-n_{y}\right)\right\}$ and $\delta\left(\xi_{k}-i\right)$ is the Kronecker Delta function.

For system (1), the following output feedback controller is designed:

$$
\mathbf{u}_{k}=\mathbf{K}_{\xi_{k}, k} \bar{y}_{k}=\mathbf{K}_{\xi_{k}, k} \Phi_{\xi_{k}} \mathbf{C}_{k} \mathbf{x}_{k}+\mathbf{K}_{\xi_{k}, k} \Phi_{\xi_{k}} \mathbf{E}_{k} \mathbf{v}_{k},
$$

where $\mathbf{K}_{\xi_{k}, k}$ is the controller gain to be designed.

According to formula (2), $\mathbf{A}_{k}\left(\mu_{k}\right)$ can be expressed as $\mathbf{A}_{k}\left(\mu_{k}\right)=\bar{A}_{k}+\widetilde{A}_{k}\left(\mu_{k}\right)$, and $\bar{w}_{k}=\left[\begin{array}{ll}\mathbf{w}_{k} & \mathbf{v}_{k}\end{array}\right]^{T}$ is defined for ease of analysis. The following closed-loop system is obtained by substituting equations (10) and (11) into system (1):

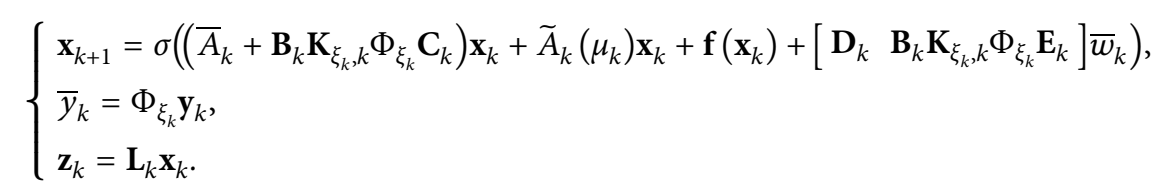

The purpose of this study is to design a finite-time domain output feedback controller (11) so that, for a given disturbance suppression level $\gamma$, positive definite matrix $\mathbf{S}$, and system initial state $\mathbf{x}_{0}$, the controlled output $\mathbf{z}_{k}$ of the system meets the following $H_{\infty}$ performance requirements:

$$
\mathscr{F}_{1} \triangleq E\left\{\sum_{k=0}^{N}\left(\left\|\mathbf{z}_{k}\right\|^{2}-\gamma^{2}\left\|\bar{w}_{k}\right\|^{2}\right)-\gamma^{2} \mathbf{x}_{0}^{T} \mathbf{S} \mathbf{x}_{0}\right\}<0, \quad \forall \bar{w}_{k} \neq 0 .
$$

\section{3. $H_{\infty}$ Performance Analysis}

System (12) contains $\mathbf{B}_{k} \mathbf{K}_{\xi_{k}, k} \Phi_{\xi_{k}} \mathbf{E}_{k} \mathbf{v}_{k}$, so to facilitate the solution of controller gain $\mathbf{K}_{\xi_{k}, k}$, we define $\bar{D}_{k}=$

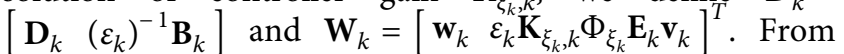
the definition of $\mathbf{W}_{k}$, perturbation $\mathbf{W}_{k} \in \mathscr{L}_{2[0, N]}$ rewrites formulae (12) to (14). The positive real number $\varepsilon_{k}$ is introduced to describe the scale of measurement interference and to provide more degrees of freedom [20] for the design of the controller:

$$
\left\{\begin{array}{l}
\mathbf{x}_{k+1}=\sigma\left(\left(\bar{A}_{k}+\mathbf{B}_{k} \mathbf{K}_{\xi_{k}, k} \Phi_{\xi_{k}} \mathbf{C}_{k}\right) \mathbf{x}_{k}+\tilde{A}_{k}\left(\mu_{k}\right) \mathbf{x}_{k}+\mathbf{f}\left(\mathbf{x}_{k}\right)+\bar{D}_{k} \mathbf{W}_{k}\right) \\
\bar{y}_{k}=\Phi_{\xi_{k}} \mathbf{y}_{k} \\
\mathbf{z}_{k}=\mathbf{L}_{k} \mathbf{x}_{k}
\end{array}\right.
$$

Next, similar to equation (13), for system (14), the following $H_{\infty}$ performance indicators are defined:

$$
\mathbf{J}_{2} \triangleq E\left\{\sum_{k=0}^{N}\left(\left\|\mathbf{z}_{k}\right\|^{2}-\gamma^{2}\left\|\mathbf{W}_{k}\right\|^{2}\right)-\gamma^{2} \mathbf{x}_{0}^{T} \mathbf{S x}_{0}\right\}<0, \quad \forall \mathbf{W}_{k} \neq 0 .
$$

Lemma 1. For performance indicators (13) and (14), if $\left(\varepsilon_{k}\right)^{2} \mathbf{E}_{k}^{T} \Phi_{\xi_{k}}^{T} \mathbf{K}_{\xi_{k}, k}^{T} \mathbf{K}_{\xi_{k}, k} \Phi_{\xi_{k}} \mathbf{E}_{k} \leq I$ and $\mathbf{J}_{2}<0$ are satisfied, then $\mathbf{J}_{1} \leq \mathbf{J}_{2}<0$.

Proof. Considering $\left(\varepsilon_{k}\right)^{2} \mathbf{E}_{k}^{T} \Phi_{\xi_{k}}^{T} \mathbf{K}_{\xi_{k}, k}^{T} \mathbf{K}_{\xi_{k}, k} \Phi_{\xi_{k}} \mathbf{E}_{k} \leq I \quad$ and $\mathbf{J}_{2}<0$, then

$$
\begin{aligned}
\mathbf{J}_{1}-\mathbf{J}_{2} & =E\left\{\sum_{k=0}^{N} \gamma^{2}\left(\left\|\mathbf{W}_{k}\right\|^{2}-\left\|\bar{w}_{k}\right\|^{2}\right)\right\} \\
& =E\left\{\sum_{k=0}^{N} \gamma^{2} \mathbf{v}_{k}^{T}\left(\left(\varepsilon_{k}\right)^{2} \mathbf{E}_{k}^{T} \Phi_{\xi_{k}}^{T} \mathbf{K}_{\xi_{k}, k}^{T} \mathbf{K}_{\xi_{k}, k} \Phi_{\xi_{k}} \mathbf{E}_{k}-I\right) \mathbf{v}_{k}\right\} \leq 0 .
\end{aligned}
$$

So, $\mathbf{J}_{1} \leq \mathbf{J}_{2}<0$. Proving is completed.

Lemma 2. For $\forall \mathbf{x}_{k} \in \mathbb{R}^{n_{x}}$, if the matrix $\mathbf{F}$ is a positive definite symmetric matrix, $\sigma^{T}\left(\mathbf{x}_{k}\right) \mathbf{F} \sigma\left(\mathbf{x}_{k}\right) \leq \lambda_{\max }\{\mathbf{F}\} \mathbf{x}_{k}^{T} \mathbf{x}_{k}$ is invariant, where $\lambda_{\max }\{\mathbf{F}\}$ represents the maximum eigenvalue of the matrix $\mathbf{F}$.

Proof. According to formula (7), $\left|\sigma_{m}\left(\mathbf{x}_{k}^{m}\right)\right| \leq\left|\mathbf{x}_{k}^{m}\right|$ can be obtained, and then, $\sigma^{T}\left(\mathbf{x}_{k}\right) \sigma\left(\mathbf{x}_{k}\right)-\mathbf{x}_{k}^{T} \mathbf{x}_{k}=\sum_{m=1}^{n_{x}}\left(\mid \sigma_{m}\right.$ $\left.\left(\mathbf{x}_{k}^{m}\right)|-| \mathbf{x}_{k}^{m} \mid\right) \leq 0$, or $\sigma^{T}\left(\mathbf{x}_{k}\right) \sigma\left(\mathbf{x}_{k}\right) \leq \mathbf{x}_{k}^{T} \mathbf{x}_{k}$ can be obtained. 
Because $\mathbf{F}$ is a positive definite symmetric matrix, then $\sigma^{T}\left(\mathbf{x}_{k}\right) \mathbf{F} \sigma\left(\mathbf{x}_{k}\right) \leq \lambda_{\max }\{\mathbf{F}\} \sigma^{T}\left(\mathbf{x}_{k}\right) \sigma\left(\mathbf{x}_{k}\right) \leq \lambda_{\max }\{\mathbf{F}\} \mathbf{x}_{k}^{T} \mathbf{x}_{k} . \quad$ The proof is completed.

Lemma 3 (see [21]). For giving nonzero matrices $\mathcal{U}, \mathscr{V}$, and $\mathscr{G}$, the optimal solution to minimize problem $\min _{\mathscr{X}}\|\boldsymbol{U} \mathscr{X} \mathscr{V}-\mathscr{G}\|_{F}$ is $\boldsymbol{U}^{\dagger} \mathscr{V} \mathscr{G}^{\dagger}$, where $\boldsymbol{U}^{\dagger}$ and $\mathscr{G}^{\dagger}$ represent the Moore-Penrose pseudoinverse of $\boldsymbol{U}$ and $\mathscr{G}$, respectively.
Theorem 1. Consider the state-saturated system (1) over finite-time domain $k \in[0, N]$ and its SCP model parameter $\xi_{k}$. Given the controller gain $\mathbf{K}_{i, k}$, positive scalar $\varepsilon_{k}, H_{\infty}$ performance index $\gamma>0$, and positive definite matrix $\mathbf{S}$, the sufficient condition for the system to meet the $H_{\infty}$ performance requirements is that the existence of positive definite symmetric matrix $\mathbf{P}_{k}^{i}$ makes the following recursive Riccatilike difference equations valid:

$$
\begin{aligned}
\mathbf{P}_{k}^{i}= & \left(\lambda_{P}\right)^{2}\left(\bar{A}_{k}+\mathbf{B}_{k} \mathbf{K}_{k} \Phi_{\xi_{k}} \mathbf{C}_{k}\right)^{T} \bar{D}_{k}\left(\Delta_{k}^{i}\right)^{-1} \bar{D}_{k}^{\mathrm{T}}\left(\bar{A}_{k}+\mathbf{B}_{k} \mathbf{K}_{i, k} \Phi_{\xi_{k}} \mathbf{C}_{k}\right)+\lambda_{P}\left(\bar{A}_{k}+\mathbf{B}_{k} \mathbf{K}_{i, k} \Phi_{\xi_{k}} \mathbf{C}_{k}\right)^{T} \\
& \left(\bar{A}_{k}+\mathbf{B}_{k} \mathbf{K}_{i, k} \Phi_{\xi_{k}} \mathbf{C}_{k}\right)+\lambda_{P} \sum_{m=1}^{n_{x}} \mathscr{T}_{a^{m, k}}+\lambda_{P} \sum_{n=1}^{q} \Gamma_{n, k} \operatorname{tr}\left(\ell_{n, k} \ell_{n, k}^{\mathrm{T}}\right)+\mathbf{L}_{k}^{T} \mathbf{L}_{k},
\end{aligned}
$$

and subjects to constraints

$$
\begin{aligned}
\Delta_{k}^{i} & >0, \\
\mathbf{P}_{0}^{i} & \leq \gamma^{2} \mathbf{S}, \\
\left(\varepsilon_{k}\right)^{2} \mathbf{E}_{k}^{T} \Phi_{\xi_{k}}^{T} \mathbf{K}_{i, k}^{T} \mathbf{K}_{i, k} \Phi_{\xi_{k}} \mathbf{E}_{k} & \leq I,
\end{aligned}
$$

where $\lambda_{P}=\lambda_{\max }\left\{\bar{P}_{k+1}^{i}\right\}, \quad \bar{P}_{k+1}^{i}=\sum_{j=1}^{n_{y}} p_{k}^{i j} \mathbf{P}_{k+1}^{j}, \quad \Delta_{k}^{i}=\gamma^{2} I-$ $\lambda_{P} \bar{D}_{k}^{T} \bar{D}_{k}$, and $\mathbf{T}_{a_{m s}^{k}, a_{m t}^{k}}$ is the $(s, t)$ element of the symmetric matrix $\mathscr{T}_{a^{m, k}}$.
Proof. Define the following function:

$$
\mathbf{J}_{k}^{i}=E\left\{\mathbf{x}_{k+1}^{T} \mathbf{P}_{k+1}^{\xi_{k+1}} \mathbf{x}_{k+1}-\mathbf{x}_{k}^{T} \mathbf{P}_{k}^{i} \mathbf{x}_{k} \mid i=\xi_{k}\right\} .
$$

According to equations (14) and Lemma 2, it can be obtained that

$$
\begin{aligned}
\mathbf{J}_{k}^{i}= & E\left\{\sigma ^ { T } ( ( \overline { A } _ { k } + \mathbf { B } _ { k } \mathbf { K } _ { i , k } \Phi _ { \xi _ { k } } \mathbf { C } _ { k } ) \mathbf { x } _ { k } + \widetilde { A } _ { k } ( \mu _ { k } ) \mathbf { x } _ { k } + \mathbf { f } ( \mathbf { x } _ { k } ) + \overline { D } _ { k } \mathbf { W } _ { k } ) \overline { \mathrm { P } } _ { k + 1 } ^ { i } \sigma \left(\left(\bar{A}_{k}+\mathbf{B}_{k} \mathbf{K}_{i, k} \Phi_{\xi_{k}} \mathbf{C}_{k}\right) \mathbf{x}_{k}+\right.\right. \\
& \left.\left.\widetilde{\mathrm{A}}_{k}\left(\mu_{k}\right) \mathbf{x}_{k}+\mathbf{f}\left(\mathbf{x}_{k}\right)+\bar{D}_{k} \mathbf{W}_{k}\right)-\mathbf{x}_{k}^{T} \mathbf{P}_{k}^{i} \mathbf{x}_{k} \mid i=\xi_{k}\right\} \\
\leq & E\left\{\lambda _ { P } \left[\mathbf{x}_{k}^{T}\left(\bar{A}_{k}+\mathbf{B}_{k} \mathbf{K}_{i, k} \Phi_{\xi_{k}} \mathbf{C}_{k}\right)^{T}\left(\bar{A}_{k}+\mathbf{B}_{k} \mathbf{K}_{i, k} \Phi_{\xi_{k}} \mathbf{C}_{k}\right) \mathbf{x}_{k}+2 \mathbf{x}_{k}^{T}\left(\bar{A}_{k}+\mathbf{B}_{k} \mathbf{K}_{i, k} \Phi_{\xi_{k}} \mathbf{C}_{k}\right)^{T} \overline{\mathrm{D}}_{k} \mathbf{W}_{k}+\right.\right. \\
& \left.\left.\mathbf{x}_{k}^{T} \widetilde{A}_{k}^{T}\left(\mu_{k}\right) \widetilde{A}_{k}\left(\mu_{k}\right) \mathbf{x}_{k}+\mathbf{f}^{T}\left(\mathbf{x}_{k}\right) \mathbf{f}\left(\mathbf{x}_{k}\right)+\mathbf{W}_{k}^{\mathrm{T}} \bar{D}_{k}^{T} \bar{D}_{k} \mathbf{W}_{k}\right]-\mathbf{x}_{k}^{T} \mathbf{P}_{k}^{i} \mathbf{x}_{k} \mid i=\xi_{k}\right\} .
\end{aligned}
$$

According to properties of matrix covariance and trace, equations (2) (5) can be obtained:

$$
\begin{aligned}
E\left\{\mathbf{x}_{k}^{T} \widetilde{A}_{k}^{T}\left(\mu_{k}\right) \widetilde{A}_{k}\left(\mu_{k}\right) \mathbf{x}_{k}\right\} & =\mathbf{x}_{k}^{T} \sum_{m=1}^{n_{x}} \mathscr{T}_{a^{m, k}} \mathbf{x}_{k}, \\
E\left\{\mathbf{f}^{T}\left(\mathbf{x}_{k}\right) \mathbf{f}\left(\mathbf{x}_{k}\right)\right\} & =\mathbf{x}_{k}^{T} \sum_{n=1}^{q} \Gamma_{n, k} \operatorname{tr}\left(\ell_{n, k} \ell_{n, k}^{T}\right) \mathbf{x}_{k} .
\end{aligned}
$$

Therefore, equation (20) is equivalent to

$$
\begin{aligned}
\mathbf{J}_{k}^{i} \leq & E\left\{x _ { k } ^ { T } \left[\lambda_{P}\left(\bar{A}_{k}+\mathbf{B}_{k} \mathbf{K}_{i, k} \Phi_{\xi_{k}} \mathbf{C}_{k}\right)^{T}\left(\bar{A}_{k}+\mathbf{B}_{k} \mathbf{K}_{i, k} \Phi_{\xi_{k}} \mathbf{C}_{k}\right)+\right.\right. \\
& \left.\lambda_{P} \sum_{m=1}^{n_{x}} \mathscr{T}_{a^{m, k}}+\lambda_{P} \sum_{n=1}^{q} \Gamma_{n, k} \operatorname{tr}\left(\ell_{n, k} \ell_{n, k}^{T}\right)-\mathbf{P}_{k}^{i}\right] \mathbf{x}_{k}+ \\
& \left.2 \lambda_{P} \mathbf{x}_{k}^{T}\left(\bar{A}_{k}+\mathbf{B}_{k} \mathbf{K}_{i, k} \Phi_{\xi_{k}} \mathbf{C}_{k}\right)^{T} \bar{D}_{k} \mathbf{W}_{k}+\lambda_{P} \mathbf{W}_{k}^{T} \bar{D}_{k}^{T} \bar{D}_{k} \mathbf{W}_{k} \mid i=\xi_{k}\right\} .
\end{aligned}
$$

Add zero item $\left\|\mathbf{z}_{k}\right\|^{2}-\gamma^{2}\left\|\mathbf{W}_{k}\right\|^{2}-\left\|\mathbf{z}_{k}\right\|^{2}+\gamma^{2}\left\|\mathbf{W}_{k}\right\|^{2}$ to the right side of equation (22), and use perfect plane method to change equation (22) into 


$$
\begin{aligned}
\mathrm{J}_{k}^{i} \leq & E\left\{\mathbf { x } _ { k } ^ { T } \left[\lambda_{P}\left(\bar{A}_{k}+\mathbf{B}_{k} \mathbf{K}_{i, k} \Phi_{\xi_{k}} \mathbf{C}_{k}\right)^{T}\left(\bar{A}_{k}+\mathbf{B}_{k} \mathbf{K}_{i, k} \Phi_{\xi_{k}} \mathbf{C}_{k}\right)+\lambda_{P} \sum_{m=1}^{n_{x}} \mathscr{T}_{a^{m, k}}+\right.\right. \\
& \left.\lambda_{P} \sum_{n=1}^{q} \Gamma_{n, k} \operatorname{tr}\left(\ell_{n, k} \ell_{n, k}^{T}\right)+\mathbf{L}_{k}^{T} \mathbf{L}_{k}-\mathbf{P}_{k}^{i}\right] \mathbf{x}_{k}+\lambda_{P} \mathbf{W}_{k}^{T} \bar{D}_{k}^{T} \bar{D}_{k} \mathbf{W}_{k}+ \\
& \left.2 \lambda_{P} \mathbf{x}_{k}^{T}\left(\bar{A}_{k}+\mathbf{B}_{k} \mathbf{K}_{i, k} \Phi_{\xi_{k}} \mathbf{C}_{k}\right)^{T} \bar{D}_{k} \mathbf{W}_{k}-\gamma^{2} \mathbf{W}_{k}^{T} \mathbf{W}_{k}-\left\|\mathbf{z}_{k}\right\|^{2}+\gamma^{2}\left\|\mathbf{W}_{k}\right\|^{2} \mid i=\xi_{k}\right\} \\
= & E\left\{\mathbf { x } _ { k } ^ { T } \left[\lambda_{P}\left(\bar{A}_{k}+\mathbf{B}_{k} \mathbf{K}_{i, k} \Phi_{\xi_{k}} \mathbf{C}_{k}\right)^{T}\left(\bar{A}_{k}+\mathbf{B}_{k} \mathbf{K}_{i, k} \Phi_{\xi_{k}} \mathbf{C}_{k}\right)+\lambda_{P} \sum_{m=1}^{n_{x}} \mathscr{T}_{a^{m, k}}+\right.\right. \\
& \left.\lambda_{P} \sum_{n=1}^{q} \Gamma_{n, k} \operatorname{tr}\left(\ell_{n, k} \ell_{n, k}^{T}\right)+\mathbf{L}_{k}^{T} \mathbf{L}_{k}-\mathbf{P}_{k}^{i}\right] \mathbf{x}_{k}+\overline{\mathbf{W}}_{k}^{T} \Delta_{k}^{i} \overline{\mathbf{W}}_{k}- \\
& \left.\left(\mathbf{W}_{k}-\overline{\mathbf{W}}_{k}\right)^{T} \Delta_{k}^{i}\left(\mathbf{W}_{k}-\overline{\mathbf{W}}_{k}\right)-\left\|\mathbf{z}_{k}\right\|^{2}+\gamma^{2}\left\|\mathbf{W}_{k}\right\|^{2} \mid i=\xi_{k}\right\}
\end{aligned}
$$

where $\overline{\mathbf{W}}_{k}=\lambda_{P}\left(\Delta_{k}^{i}\right)^{-1} \bar{D}_{k}^{T}\left(\bar{A}_{k}+\mathbf{B}_{k} \mathbf{K}_{i, k} \Phi_{\xi_{k}} \mathbf{C}_{k}\right) \mathbf{x}_{k}$.

$$
\begin{aligned}
& E\left\{\mathbf{x}_{N+1}^{T} \mathbf{P}_{N+1}^{\xi_{N+1}} \mathbf{x}_{N+1}-\mathbf{x}_{0}^{T} \mathbf{P}_{0}^{i} \mathbf{x}_{0}\right\} \\
\leq & E\left\{-\sum_{k=0}^{N}\left(\mathbf{W}_{k}-\overline{\mathbf{W}}_{k}\right)^{T} \Delta_{k}^{i}\left(\mathbf{W}_{k}-\overline{\mathbf{W}}_{k}\right)-\sum_{k=0}^{N}\left(\left\|\mathbf{z}_{k}\right\|^{2}-\gamma^{2}\left\|\mathbf{W}_{k}\right\|^{2}\right)\right\} .
\end{aligned}
$$

According to the properties of conditional expectation, inequality (23) is accumulated from 0 to $N$, and the difference equation (17) such as Riccati is obtained
Considering $\Delta_{k}^{i}>0, \quad \mathbf{P}_{0}^{i} \leq \gamma^{2} \mathbf{S}, \quad\left(\varepsilon_{k}\right)^{2} \mathbf{E}_{k}^{T} \Phi_{\xi_{k}}^{T} \mathbf{K}_{i, k}^{T} \mathbf{K}_{i, k} \Phi_{\xi_{k}}$ $\mathbf{E}_{k} \leq I$, and the matrix $\mathbf{P}_{k+1}^{i}$ is positively definite symmetry, it is not difficult to obtain

$$
\mathbf{J}_{2} \leq E\left\{\mathbf{x}_{k}^{T}\left(\mathbf{P}_{0}^{i}-\gamma^{2} \mathbf{S}\right) \mathbf{x}_{k}-\sum_{k=0}^{N}\left(\mathbf{W}_{k}-\overline{\mathbf{W}}_{k}\right)^{T} \Delta_{k}^{i}\left(\mathbf{W}_{k}-\overline{\mathbf{W}}_{k}\right)\right\}<0 .
$$

Therefore, $\mathbf{J}_{1} \leq \mathbf{J}_{2}<0$; the system meets the $H_{\infty}$ performance requirements. Proof is completed.

\section{4. $H_{\infty}$ Controller Design}

Theorem 1 gives a sufficient condition for the closed-loop system (1) to satisfy the $H_{\infty}$ performance index. Next, the solution of controller gain matrix $\mathbf{K}_{i, k}$ under the worst disturbance case of the system is $\mathbf{W}_{k}=\overline{\mathbf{W}}_{k}=\lambda_{P}\left(\Delta_{k}^{i}\right)^{-1}$ $\bar{D}_{k}^{T}\left(\bar{A}_{k}+\mathbf{B}_{k} \mathbf{K}_{i, k} \Phi_{\xi_{k}} \mathbf{C}_{k}\right) \mathbf{x}_{k}$. To do this, define $\mathscr{F}_{k}=\lambda_{P}\left(\Delta_{k}^{i}\right)^{-1}$ $\bar{D}_{k}^{T}\left(\bar{A}_{k}+\mathbf{B}_{k} \mathbf{K}_{i, k} \Phi_{\xi_{k}} \mathbf{C}_{k}\right), \quad \bar{u}_{k}=\mathbf{K}_{i, k} \Phi_{\xi_{k}} \mathbf{C}_{k} \mathbf{x}_{k}$, and the cost function $\mathbf{J}_{\overline{\mathbf{W}}_{k}} \triangleq E\left\{\sum_{k=0}^{\xi_{k}}\left(\left\|\mathbf{z}_{k}\right\|^{2}+\left\|\bar{u}_{k}\right\|^{2}\right)\right\}$. Rewrite the closedloop system (14) as

$$
\left\{\begin{array}{l}
\mathbf{x}_{k+1}=\sigma\left(\left(\bar{A}_{k}+\mathbf{B}_{k} \mathbf{K}_{i, k} \Phi_{\xi_{k}} \mathbf{C}_{k}\right) \mathbf{x}_{k}+\widetilde{A}_{k}\left(\mu_{k}\right) \mathbf{x}_{k}+\mathbf{f}\left(\mathbf{x}_{k}\right)+\bar{D}_{k} \mathscr{F}_{k} \mathbf{x}_{k}\right) \\
\bar{y}_{k}=\Phi_{\xi_{k}} \mathbf{y}_{k} \\
\mathbf{z}_{k}=\mathbf{L}_{k} \mathbf{x}_{k} .
\end{array}\right.
$$

Theorem 2. Considering the state saturation system (1) and its SCP model parameter $\xi_{k}$ on finite-time domain $k \in[0, N]$ and given the positive scalar $\varepsilon_{k}, H_{\infty}$ performance index $\gamma>0$, and positive definite matrix $\mathbf{S}$, the sufficient condition for the system to meet the $H_{\infty}$ performance requirements is that there are $\mathbf{K}_{i, k}$ and positive definite symmetric matrices $\mathbf{P}_{k}^{i}$ and $\mathbf{Q}_{k}^{i}$, so the Riccati-like difference equation recursively can be established as follows: 


$$
\begin{aligned}
\mathrm{P}_{k}^{i}= & \left(\lambda_{P}\right)^{2}\left(\bar{A}_{k}+\mathbf{B}_{k} \mathbf{K}_{i, k} \Phi_{\xi_{k}} \mathbf{C}_{k}\right)^{T} \bar{D}_{k}\left(\Delta_{k}^{i}\right)^{-1} \bar{D}_{k}^{T}\left(\bar{A}_{k}+\mathbf{B}_{k} \mathbf{K}_{i, k} \Phi_{\xi_{k}} \mathbf{C}_{k}\right)+\lambda_{P}\left(\bar{A}_{k}+\mathbf{B}_{k} \mathbf{K}_{i, k} \Phi_{\xi_{k}} \mathbf{C}_{k}\right)^{T} . \\
& \left(\bar{A}_{k}+\mathbf{B}_{k} \mathbf{K}_{i, k} \Phi_{\xi_{k}} \mathbf{C}_{k}\right)+\lambda_{P} \sum_{m=1}^{n_{x}} \mathscr{T}_{a^{m, k}}+\lambda_{P} \sum_{n=1}^{q} \Gamma_{n, k} \operatorname{tr}\left(\ell_{n, k} \ell_{n, k}^{T}\right)+\mathbf{L}_{k}^{T} \mathbf{L}_{k} \\
\mathbf{Q}_{k}^{i}= & -\left(\lambda_{Q}\right)^{2}\left(\bar{A}_{k}+\bar{D}_{k} \mathscr{F}_{k}\right)^{T} \mathbf{B}_{k}\left(\bar{\Delta}_{k}^{i}\right)^{-1} \mathbf{B}_{k}^{T}\left(\bar{A}_{k}+\bar{D}_{k} \mathscr{F}_{k}\right)+\lambda_{Q}\left(\bar{A}_{k}+\bar{D}_{k} \mathscr{F}_{k}\right)^{T} . \\
& \left(\bar{A}_{k}+\bar{D}_{k} \mathscr{F}_{k}\right)+\lambda_{Q} \sum_{m=1}^{n_{x}} \mathscr{T}_{a^{m, k}}+\lambda_{Q} \sum_{n=1}^{q} \Gamma_{n, k} \operatorname{tr}\left(\ell_{n, k} \ell_{n, k}^{T}\right)+\mathbf{L}_{k}^{T} \mathbf{L}_{k} .
\end{aligned}
$$

And, it subjects to constraints

$$
\begin{aligned}
\Delta_{k}^{i} & >0, \\
\bar{\Delta}_{k}^{i} & >0, \\
\mathbf{P}_{0}^{i} & \leq \gamma^{2} \mathbf{S}, \\
\left(\varepsilon_{k}\right)^{2} \mathbf{E}_{k}^{T} \Phi_{\xi_{k}}^{T} \mathbf{K}_{i, k}^{T} \mathbf{K}_{i, k} \Phi_{\xi_{k}} \mathbf{E}_{k} & \leq I .
\end{aligned}
$$

At this point, the gain matrix of the controller can be calculated by the following formula:

$$
\mathbf{K}_{i, k}=\mathscr{M}_{k}^{\dagger} \mathcal{N}_{k}\left(\Phi_{\xi_{k}} \mathbf{C}_{k}\right)^{\dagger}
$$

$$
\begin{aligned}
\bar{J}_{k}^{i}= & E\left\{\sigma ^ { T } ( ( \overline { A } _ { k } + \overline { D } _ { k } \mathscr { F } _ { k } ) \mathbf { x } _ { k } + \widetilde { A } _ { k } ( \mu _ { k } ) \mathbf { x } _ { k } + \mathbf { B } _ { k } \mathbf { K } _ { i , k } \Phi _ { \xi _ { k } } \mathbf { C } _ { k } \mathbf { x } _ { k } + \mathbf { f } ( \mathbf { x } _ { k } ) ) \overline { Q } _ { k + 1 } ^ { i } \sigma \left(\left(\bar{A}_{k}+\bar{D}_{k} \mathscr{F}_{k}\right) \mathbf{x}_{k}+\right.\right. \\
& \left.\left.\widetilde{A}_{k}\left(\mu_{k}\right) \mathbf{x}_{k}+\mathbf{B}_{k} \mathbf{K}_{i, k} \Phi_{\xi_{k}} \mathbf{C}_{k} \mathbf{x}_{k}+\mathbf{f}\left(\mathbf{x}_{k}\right)\right)-\mathbf{x}_{k}^{T} \mathbf{Q}_{k}^{i} \mathbf{x}_{k} \mid i=\xi_{k}\right\} \\
\leq & E\left\{\mathbf { x } _ { k } ^ { T } \left[\lambda_{Q}\left(\bar{A}_{k}+\bar{D}_{k} \mathscr{F}_{k}\right)^{T}\left(\bar{A}_{k}+\bar{D}_{k} \mathscr{F}_{k}\right)+\lambda_{Q} \sum_{m=1}^{n_{x}} \mathscr{T}_{a^{m, k}}+\right.\right. \\
& \left.\lambda_{Q} \sum_{n=1}^{q} \Gamma_{n, k} \operatorname{tr}\left(\ell_{n, k} \ell_{n, k}^{T}\right)-\mathbf{Q}_{k}^{i}\right] \mathbf{x}_{k}+\lambda_{Q} \mathbf{x}_{k}^{T} \mathbf{C}_{k}^{T} \Phi_{\xi_{k}}^{T} \mathbf{K}_{i, k}^{T} \mathbf{B}_{k}^{T} \mathbf{B}_{k} \mathbf{K}_{i, k} \Phi_{\xi_{k}} \mathbf{C}_{k} \mathbf{x}_{k}+ \\
& \left.2 \lambda_{Q} \mathbf{x}_{k}^{T}\left(\overline{\mathrm{A}}_{k}+\overline{\mathrm{D}}_{k} \mathscr{F}_{k}\right)^{T} \mathbf{B}_{k} \mathbf{K}_{i, k} \Phi_{\xi_{k}} \mathbf{C}_{k} \mathbf{x}_{k} \mid i=\xi_{k}\right\} .
\end{aligned}
$$

Add the zero term $E\left\{\sum_{k=0}^{N} \bar{J}_{k}^{i}-\sum_{k=0}^{N} \bar{J}_{k}^{i}\right\}$ to the right of the cost function $\mathbf{J}_{\overline{\mathbf{W}}_{k}}$, then define $\tilde{u}_{k}=-\lambda_{Q}$ $\left(\bar{\Delta}_{k}^{i}\right)^{-1} \mathbf{B}_{k}^{T}\left(\bar{A}_{k}+\bar{D}_{k} \mathscr{F}_{k}\right) \mathbf{x}_{k}$, use the complete square for $\bar{u}_{k}$,

and combine equation (27) and constraint (28), and we obtain

$$
\begin{aligned}
\mathbf{J}_{\overline{\mathbf{W}}_{k}} & =E\left\{\sum_{k=0}^{N}\left(\left\|\mathbf{z}_{k}\right\|^{2}+\left\|\bar{u}_{k}\right\|^{2}+\bar{J}_{k}^{i}\right)+\mathbf{x}_{k}^{T} \mathbf{Q}_{0}^{i} \mathbf{x}_{k}-\mathbf{x}_{N+1}^{T} \mathbf{Q}_{N+1}^{\xi(N+1)} \mathbf{x}_{N} \mid i=\xi_{k}\right\} \\
& \leq E\left\{\sum_{k=0}^{N}\left(\bar{u}_{k}-\widetilde{u}_{k}\right)^{T} \bar{\Delta}_{k}^{i}\left(\bar{u}_{k}-\widetilde{u}_{k}\right)\right\}+E\left\{\mathbf{x}_{k}^{T} \mathbf{Q}_{0}^{i} \mathbf{x}_{k}\right\} \\
& \leq E\left\{\sum_{k=0}^{N}\left\|\mathbf{K}_{i, k} \Phi_{\xi_{k}} \mathbf{C}_{k}+\lambda_{Q}\left(\Delta_{k}^{i}\right)^{-1} \mathbf{B}_{k}^{T}\left(\bar{A}_{k}+\bar{D}_{k} \mathscr{F}_{k}\right)\right\|_{F}^{2}\left\|\bar{\Delta}_{k}^{i}\right\|_{F}^{2}\left\|\mathbf{x}_{k}\right\|^{2}\right\}+E\left\{\mathbf{x}_{k}^{T} \mathbf{Q}_{0}^{i} \mathbf{x}_{k}\right\} .
\end{aligned}
$$


Step 1. Set $k=N$, for all $i \in\left\{1,2, \ldots, n_{y}\right\}$; given disturbance suppression level $\gamma>0$ positive definite matrices $\mathbf{S}, \mathbf{P}_{N+1}^{i}$ and $\mathbf{Q}_{N+1}^{i}$ are appropriate positive definite symmetric matrices.

Step 2. Calculate $\Delta_{k}^{i}$ and $\bar{\Delta}_{k}^{i}$. If $\Delta_{k}^{i}>0$ and $\bar{\Delta}_{k}^{i}>0$, go to the next step, otherwise go to Step 6 .

Step 3. $\mathscr{M}_{k}$ and $\mathcal{N}_{k}$ were calculated, and controller gain matrix $\mathbf{K}_{i, k}$ was solved according to equation (29). If $\left(\varepsilon_{k}\right)^{2} \mathbf{E}_{k}^{T} \Phi_{\xi_{k}}^{T} \mathbf{K}_{i, k}^{T} \mathbf{K}_{i, k} \Phi_{\xi_{k}} \mathbf{E}_{k} \leq I$, go to the next step, otherwise go to Step6.

Step 4. $\mathbf{P}_{k}^{i}$ and $\mathbf{Q}_{k}^{i}$ were obtained by solving Riccati-like difference equation (27), and then, $\lambda_{P}$ and $\lambda_{Q}$ were obtained. If $\mathbf{P}_{k}^{i}>0$ and $\mathbf{Q}_{k}^{i}>0$, go to the next step, otherwise go to Step 6 .

Step 5. If $k \neq 0$, make $k=k-1$ and go to Step2; otherwise, judge whether $\mathbf{P}_{0}^{i} \leq \gamma^{2} \mathbf{S}$ is established; then, the calculation terminates; if not, then proceed to the next step.

Step 6. The algorithm is unsolvable and the calculation is terminated.

Algorithm 1: $H_{\infty}$ controller design algorithm on finite time domain $[0, N]$.

It is noted that $\mathscr{F}_{k}=\lambda_{P}\left(\Delta_{k}^{i}\right)^{-1} \bar{D}_{k}^{T}\left(\bar{A}_{k}+\mathbf{B}_{k} \mathbf{K}_{i, k} \Phi_{\xi_{k}} \mathbf{C}_{k}\right)$, in order to reduce the cost function, the gain matrix of the controller is selected as follows:

$$
\begin{aligned}
\mathbf{K}_{i, k} & =\arg \min _{\mathbf{K}_{i, k}}\left\|\mathbf{K}_{i, k} \Phi_{\xi_{k}} \mathbf{C}_{k}+\lambda_{Q}\left(\Delta_{k}^{i}\right)^{-1} \mathbf{B}_{k}^{T}\left(\bar{A}_{k}+\bar{D}_{k} \mathscr{F}_{k}\right)\right\|_{F} \\
& =\arg \min _{\mathbf{K}_{i, k}}\left\|\mathscr{M}\left(\mathbf{K}_{i, k} \Phi_{\xi_{k}} \mathbf{C}_{k}\right)-\mathcal{N}_{k}\right\| .
\end{aligned}
$$

Therefore, according to Lemma 3, equation (29) is the optimal solution to the minimization problem (33). Proving is complete.

Based on Theorem 2, the following $H_{\infty}$ controller design algorithm on finite-time domain $[0, N]$ is obtained (Algorithm 1).

\section{Numerical Example}

A state-saturated time-varying system (1) with random parameters and random nonlinearity is considered in finitetime domain $k \in[0,30]$, and its system matrix parameters are shown as below [22]:

$$
\begin{aligned}
\mathbf{A}_{k}\left(\mu_{k}\right) & =\bar{A}_{k}+\widetilde{A}_{k}\left(\mu_{k}\right) \\
& =\left[\begin{array}{cc}
0.42+\sin (2 k-1) & -0.4 \\
-0.4+e^{-5 k} & 0.85
\end{array}\right]+\mu_{k}\left[\begin{array}{cc}
0.1 & 0 \\
0 & 0.2
\end{array}\right], \\
\mathbf{B}_{k} & =\left[\begin{array}{c}
0.85 \\
-0.65
\end{array}\right], \\
\mathbf{C}_{k} & =\left[\begin{array}{cc}
0.65 & -0.7 \\
-0.6 & 0.65
\end{array}\right], \\
\mathbf{D}_{k} & =\left[\begin{array}{ll}
-0.02 & 0.015
\end{array}\right]^{T}, \\
\mathbf{E}_{k} & =\left[\begin{array}{ll}
0.01 & 0.01
\end{array}\right]^{T}, \\
\mathbf{L}_{k} & =\left[\begin{array}{ll}
0.2 & 0.2
\end{array}\right] .
\end{aligned}
$$

The random nonlinear function is

$$
\mathbf{f}\left(\mathbf{x}_{k}\right)=\left(0.1 \operatorname{sign}\left(x_{k}^{1}\right) x_{k}^{1} \bar{\mu}_{1 k}+0.2 \operatorname{sign}\left(x_{k}^{2}\right) x_{k}^{2} \sin \left(\bar{\mu}_{2 k}\right)\right)\left[\begin{array}{l}
0.06 \\
0.09
\end{array}\right]
$$

where $\mu_{k}, \bar{\mu}_{1 k}$, and $\bar{\mu}_{2 k}$ are three Gaussian white noise sequences whose mean value is 0 and variance is 1 , and it is not difficult to verify that the random nonlinear function satisfies

$$
\begin{aligned}
\mathbf{E}\left\{\mathbf{f}\left(\mathbf{x}_{k}\right) \mid \mathbf{x}_{k}\right\} & =0, \\
\mathbf{E}\left\{\mathbf{f}\left(\mathbf{x}_{k}\right) \mathbf{f}^{T}\left(\mathbf{x}_{k}\right) \mid \mathbf{x}_{k}\right\} & =\left[\begin{array}{l}
0.06 \\
0.09
\end{array}\right]\left[\begin{array}{l}
0.06 \\
0.09
\end{array}\right]^{T} \mathbf{x}_{k}^{T}\left[\begin{array}{cc}
0.01 & 0 \\
0 & 0.0173
\end{array}\right] \mathbf{x}_{k} .
\end{aligned}
$$

In addition, it is known that the initial state $\mathbf{x}_{0}=\left[\begin{array}{ll}0.5 & 0.1\end{array}\right]^{T}, \mathbf{x}_{k, \max }^{1}, \mathbf{x}_{k, \max }^{2}$, and $\varepsilon_{k}$ of the system is 1 , the state transition matrix of the random communication protocol $P_{k}=\left[\begin{array}{cc}0.4+0.1(-1)^{k} & 0.6-0.1(-1)^{k} \\ 0.75 & 0.25\end{array}\right]$, and the process noise and measurement interference of the system are $\mathbf{w}_{k}=1.5 \sin (k)$ and $\mathbf{v}_{k}=0.7 \cos (0.7 k)$, respectively.

On the premise that the algorithm in this paper has a solution and the $H_{\infty}$ performance index is as small as possible, the given values of $\gamma=0.92$, positive definite matrix $\mathbf{S}=0.2 I$, and $\mathbf{P}_{N+1}^{i}$ and $\mathbf{Q}_{N+1}^{i}$ are given as 0.01I. By using the algorithm proposed in this paper, the gain matrix of the controller is obtained, as shown in Table 1. The corresponding simulation results of the system are shown in Figures 1-3. Figure 1 depicts the changes of sensor nodes that obtain network permissions under the influence of random communication protocol with the state transition matrix. Figures 2 and 3, respectively, show the state response curves of the open-loop system and the closed-loop system. The simulation results demonstrate the effectiveness of the proposed algorithm for the finite-time domain controller design.

In order to highlight the advantages of the controller design algorithm proposed in this paper in the aspect of state saturation system, the algorithm in [22] is compared with the algorithm proposed in this paper. Regardless of random parameters and random nonlinearity, only for the state saturation system under the influence of random communication protocol, the parameters set are exactly the same as those in the above simulation. It is worth noting that the algorithm in [22] is not applicable to networked control systems under the influence of random communication protocols, so $P_{k}$ and $Q_{k}$ in [22] need to be adjusted. The controller gain matrix $K_{i, k}$ obtained according to the 
TABLE 1: Gain matrix of $H_{\infty}$ controller in finite-time domain.

\begin{tabular}{|c|c|c|c|c|c|}
\hline$k$ & 0 & 1 & 2 & 3 & 4 \\
\hline$\overline{\mathbf{K}_{i, k}}$ & {$\left[\begin{array}{ll}-0.0779 & 0\end{array}\right]$} & {$\left[\begin{array}{ll}0 & 0.7157\end{array}\right]$} & {$\left[\begin{array}{ll}-0.5168 & 0\end{array}\right]$} & {$\left[\begin{array}{ll}-0.259 & 0\end{array}\right]$} & {$\left[\begin{array}{ll}-0.6535 & 0\end{array}\right]$} \\
\hline$k^{l, n}$ & 5 & 6 & $\ldots$ & 29 & 30 \\
\hline $\mathbf{K}_{i, k}$ & {$\left[\begin{array}{ll}-0.5704 & 0\end{array}\right]$} & {$\left[\begin{array}{ll}0 & 0.2589\end{array}\right]$} & $\cdots$ & {$\left[\begin{array}{ll}0 & 0.1248\end{array}\right]$} & {$\left[\begin{array}{ll}-0.0151 & 0\end{array}\right]$} \\
\hline
\end{tabular}

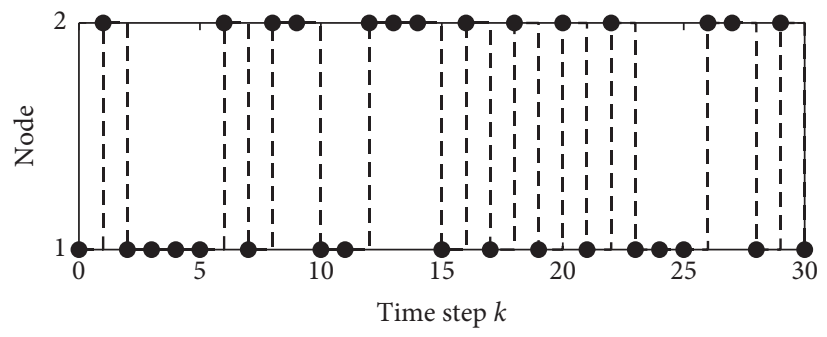

Selected nodes $\xi_{k}$

Figure 1: Changes of the selected sensor nodes.

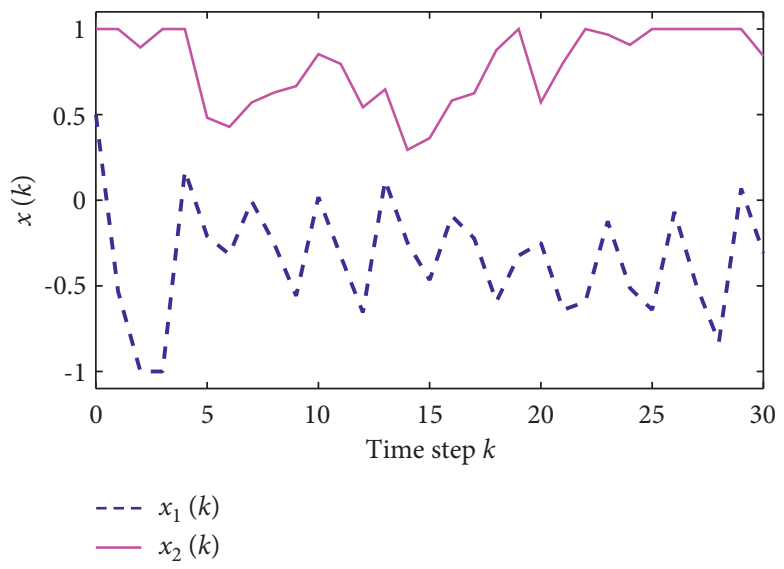

Figure 2: The state trajectories of the open-loop system with state saturation.

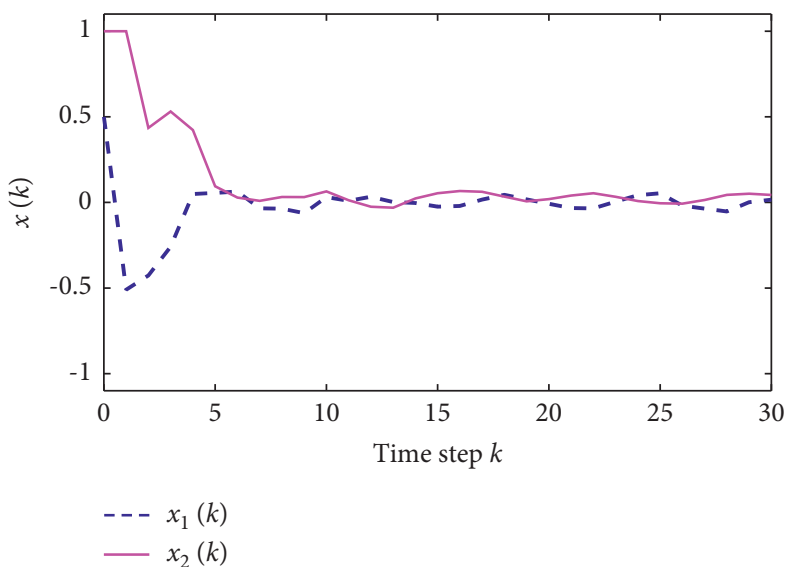

FIgURE 3: The state trajectories of the closed-loop system with state saturation.

algorithm proposed in this paper and the controller gain matrix $K_{i, k}^{*}$ obtained according to the algorithm in [22] are shown in Table 2. The state response curves of the corresponding closed-loop system are shown in Figures 4 and 5, respectively. It can be seen from Figure 4 that the controller gain matrix $K_{i, k}$ obtained based on the algorithm 
TABLE 2: Gain matrix of finite-time domain $H_{\infty}$ controller obtained by two algorithms.

\begin{tabular}{|c|c|c|c|c|c|}
\hline$k$ & 0 & 1 & 2 & 3 & 4 \\
\hline$\overline{\mathbf{K}_{i, k}}$ & {$\left[\begin{array}{ll}-0.0793 & 0\end{array}\right]$} & {$\left[\begin{array}{ll}0 & 0.7189\end{array}\right]$} & {$\left[\begin{array}{ll}-0.5201 & 0\end{array}\right]$} & {$\left[\begin{array}{ll}-0.2631 & 0\end{array}\right]$} & {$\left[\begin{array}{ll}-0.6605 & 0\end{array}\right]$} \\
\hline $\mathbf{K}_{i, k}^{*, \kappa}$ & {$\left[\begin{array}{ll}-0.0478 & 0\end{array}\right]$} & {$\left[\begin{array}{ll}0 & 0.3120\end{array}\right]$} & {$\left[\begin{array}{ll}-0.1680 & 0\end{array}\right]$} & {$\left[\begin{array}{ll}-0.1779 & 0\end{array}\right]$} & {$\left[\begin{array}{lll}-0.3010 & 0\end{array}\right]$} \\
\hline$k$ & 5 & 6 & $\ldots$ & 29 & 30 \\
\hline $\mathbf{K}_{i, k}$ & {$\left[\begin{array}{ll}-0.5716 & 0\end{array}\right]$} & {$\left[\begin{array}{ll}0 & 0.2619\end{array}\right]$} & $\cdots$ & {$\left[\begin{array}{ll}0 & 0.1270\end{array}\right]$} & {$\left[\begin{array}{ll}-0.0151 & 0\end{array}\right]$} \\
\hline $\mathbf{K}_{i, k}^{*}$ & {$\left[\begin{array}{ll}-0.1809 & 0\end{array}\right]$} & {$\left[\begin{array}{ll}0 & 0.1970\end{array}\right]$} & $\cdots$ & {$\left[\begin{array}{ll}0 & -0.0001\end{array}\right]$} & {$\left[\begin{array}{ll}0 & 0\end{array}\right]$} \\
\hline
\end{tabular}

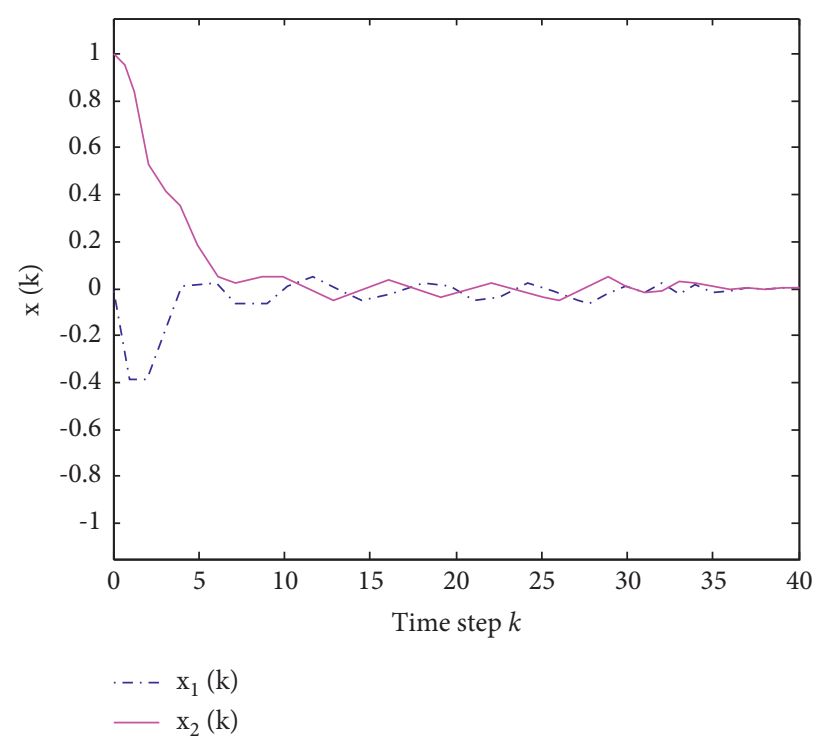

FIgURE 4: The state trajectories of the state-saturated system with gain matrix $\mathbf{K}_{i, k}$.

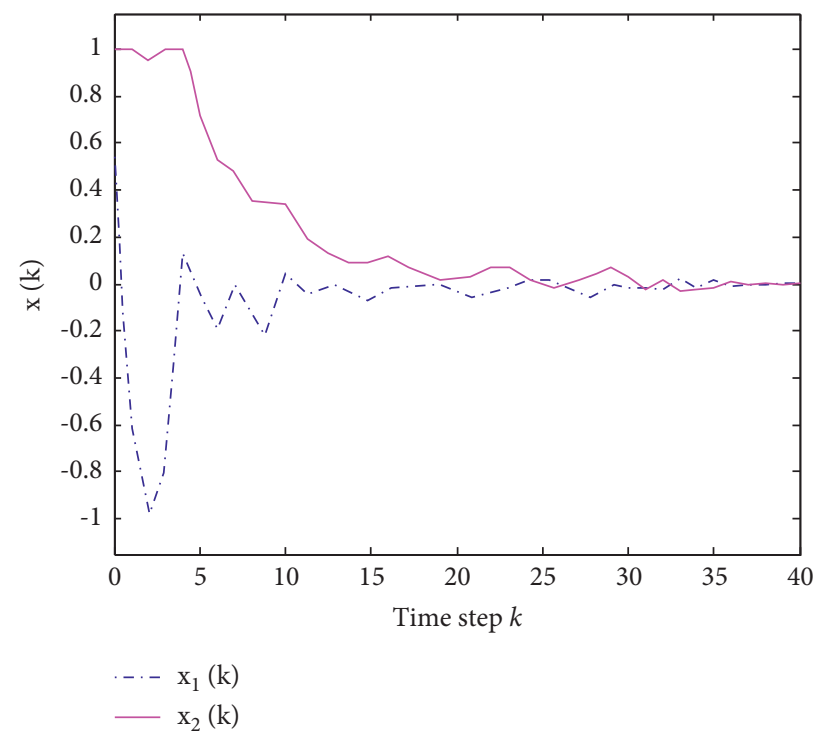

FIgURE 5: The state trajectories of the state-saturated system with gain matrix $\mathbf{K}_{i, k}^{*}$.

proposed in this paper makes the state-saturated system enter a stable state at the 6th transmission moment. It can be seen from Figure 5 that the controller gain matrix $K_{i, k}^{*}$ obtained from [22] makes the system enter a stable state at the 20th transmission moment. For this system, other parameters remain unchanged. On the premise that the controller has a solution, $\gamma$ in this algorithm can be taken as the minimum $\gamma=0.88$, and in [22], the minimum $\gamma$ can be 
taken as $\gamma=0.80$. To sum up, for the state saturation system under the influence of random communication protocol, the algorithm proposed in this paper takes into account many system factors, and the value of $\gamma$ is relatively large, but the dynamic performance of the system is better.

\section{Conclusion}

In this paper, the finite-time domain $H_{\infty}$ control problem of state-saturated time-varying systems with random parameters and random nonlinearity under the influence of random communication protocol is studied. Firstly, a state saturation system model with random parameters and random nonlinearity is constructed. Then, in the sensorcontroller transmission network, random communication protocol is added to avoid data conflict. Then, the sufficient conditions for the system to meet the $H_{\infty}$ performance requirements are obtained by using the model transformation technology and matching method. On this basis, the controller design algorithm based on the reverse-recursive Riccati difference equation technology is presented. Finally, numerical simulation shows the effectiveness of the proposed algorithm.

\section{Data Availability}

The data used to support the findings of this study are available within the article as references and from the corresponding author upon request.

\section{Conflicts of Interest}

The authors declare that they have no conflicts of interest.

\section{Acknowledgments}

This work was supported in part by the National Natural Science Foundation of China (no. 61773182).

\section{References}

[1] D. Zhang, Q. G. Wang, Y. Li, and Q.-K. Shao, “\$H_linfty\$ filtering for networked systems with multiple time-varying transmissions and random packet dropouts," IEEE Transactions on Industrial Informatics, vol. 9, no. 3, pp. 1705-1716, 2013.

[2] M. Lješnjanin, N. Dragan, and E. Daniel, "Robust stability of a class of networked control systems," Automatica, vol. 73, pp. 117-124, 2016.

[3] Y. Wu and R. Lu, "Event-based control for network systems via integral quadratic constraints," IEEE Transactions on Circuits and Systems I: Regular Papers, vol. 65, no. 4, pp. 1386-1394, 2018.

[4] R. H. Xie, W. H. Fan, C. Wang, and Q. Chen, "Dead band scheduling and control co-design method for uncertain networked control systems," Journal of Nanjing University of Science and Technology, vol. 40, no. 6, pp. 643-652, 2016, in Chinese.

[5] H. Li, H. Liu, H. Gao, and P. Shi, "Reliable fuzzy control for active suspension systems with actuator delay and fault," IEEE Transactions on Fuzzy Systems, vol. 20, no. 2, pp. 342-357, 2012.
[6] J. Liang, F. Sun, and X. Liu, "Finite-horizonHoofiltering for time-varying delay systems with randomly varying nonlinearities and sensor saturations," Systems Science \& Control Engineering, vol. 2, no. 1, pp. 108-118, 2014.

[7] L. Ma, Z. Wang, Q.-L. Han, and H.-K. Lam, "Envelopeconstrained $\mathrm{H} \infty$ filtering for nonlinear systems with quantization effects: the finite horizon case," Automatica, vol. 93, pp. 527-534, 2018.

[8] D. W. Zhang, Q. L. Han, and X. C. Jia, "Network-based output tracking control for a class of T-S fuzzy systems that can not be stabilized by nondelayed output feedback controllers," IEEE Transactions on Cybernetics, vol. 45, no. 8, pp. 15111524, 2017.

[9] C. Ahmet, I. Hideaki, and H. Tomohisa, "Networked control under random and malicious packet losses," IEEE Transactions on Automatic Control, vol. 62, no. 5, pp. 2434-2449, 2017.

[10] L. Yu, R. Y. Ling, and D. Zhang, "Ho filtering for a class of networked systems with stochastic sampling," Control and Decision, vol. 29, no. 6, pp. 1035-1040, 2014, in Chinese.

[11] D. Zhang and Y. Liu, "Fault estimation for complex networks with model uncertainty and stochastic communication protocol," Systems Science \& Control Engineering, vol. 7, no. 1, pp. 45-53, 2019.

[12] P.-d. Zhou, L. Yu, H. B. Song, and L.-1. Ou, "Ho filtering for network-based systems with stochastic protocols," Control Theory and Applications, vol. 27, no. 12, pp. 1711-1716, 2010, in Chinese.

[13] F. E. Alsaadi, Y. Luo, Y. Liu, and Z. Wang, "State estimation for delayed neural networks with stochastic communication protocol: the finite-time case," Neurocomputing, vol. 281, pp. 86-95, 2018.

[14] J. Zhang, C. Peng, X. P. Xie, and D. Yue, "Output feedback stabilization of networked control systems under a stochastic scheduling protocol," IEEE transactions on cybernetics, vol. 50, pp. 2851-2860, 2019.

[15] J. Palraj, K. Mathiyalagan, and P. Shi, "New results on robust sliding mode control for linear time-delay systems," IMA Journal of Mathematical Control and Information, vol. 38, no. 1, pp. 320-336, 2020.

[16] K. Mathiyalagan and G. Sangeetha, "Finite-time stabilization of nonlinear time delay systems using LQR based sliding mode control," Journal of the Franklin Institute, vol. 356, no. 7, pp. 3948-3964, 2019.

[17] X. F. Ji and X. L. Zhu, "Stability analysis and controller synthesis for singular discrete linear systems with state saturation," Information and Control, vol. 42, no. 4, pp. 437-442, 2013, in Chinese.

[18] C. Wen, Z. Wang, Q. Liu, and F. E. Alsaadi, "Recursive distributed filtering for a class of state-saturated systems with fading measurements and quantization effects," IEEE Transactions on Systems, Man, and Cybernetics: Systems, vol. 48, no. 6, pp. 930-941, 2018.

[19] L. Wang, Z. Wang, Q.-L. Han, and G. Wei, "Event-based variance-constrained $\$\{\backslash \text { mathcal }\{\mathrm{H}\}\}_{-}\{$linfty $\} \$$ filtering for stochastic parameter systems over sensor networks with successive missing measurements," IEEE transactions on cybernetics, vol. 48, no. 3, pp. 1007-1017, 2018.

[20] Y. S. Hung and F. W. Yang, "Robust Ho filtering for discrete time-varying uncertain systems with a known deterministic input," International Journal of Control, vol. 75, no. 15, pp. 1159-1169, 2002.

[21] R. Penrose and J. A. Todd, "On best approximate solutions of linear matrix equations," Mathematical Proceedings of the 
Cambridge Philosophical Society, vol. 52, no. 1, pp. 17-19, 1956.

[22] D. Ding, Z. Wang, J. Lam, and B. Shen, "Finite-horizon \$ \{ \cal $\mathrm{H}\}_{-}\{$infty\}\$ control for discrete time-varying systems with randomly occurring nonlinearities and fading measurements," IEEE Transactions on Automatic Control, vol. 60, no. 9, pp. 2488-2493, 2015. 